\title{
Cavernous Vessels Smooth Muscle Layer Thickness Correlates with Myocardium Collagen Deposition in High-Fat Fed Rats
}

\author{
I. Tomada*, D. Fernandes*, N. Tomada**, H. Almeida* and D. Neves* \\ * Department of Experimental Biology, Faculty of Medicine and IBMC of Universidade do Porto - \\ 4200-319 Porto, Portugal \\ ** Department of Urology of Centro Hospitalar S. João, EPE - 4200-319 Porto, Portugal
}

Vascular aging is associated with progressive deterioration of vascular homeostasis, a phenomenon that affects organs in different extent. The small vessels of the corpus cavernosum (CC) are very sensitive to functional and structural changes, and particularly susceptible to age-related modifications and atherosclerotic occlusion [1]. Penile erection is a vascular event largely regulated by the relaxation of arterial and corporal smooth muscle (SM) [2] that directly relates to its content and integrity, and inversely to that of connective tissue (CT) [3]. Corpora expansion also depends on nitric oxide (NO) bioavailability, a molecule with recognized vasodilatory and antifibrotic properties. Thus, endothelial dysfunction mainly provoked by NO loss is currently considered equivalent to vasculogenic erectile dysfunction (ED) [4], and the earliest clinical manifestation of atherosclerosis and systemic vascular disease [5]. Collagen deposition in tissues, such as $\mathrm{CC}$ and heart, strongly compromises their function and is one of the causes of ED and cardiac failure, respectively [6], but as far as we know no correlation between peripheral and cardiac endothelial dysfunction has been already demonstrated. Therefore, we aimed to evaluate morphological structure and collagen deposition in rat $\mathrm{CC}$, myocardium and tail artery during aging, under high-fat diet (HFD) consumption (a strong contributor to endothelial dysfunction onset), and energy restriction.

Male Sprague-Dawley rats (2 months-old), individually housed with free access to tap water and maintained under controlled conditions, were randomly divided into three experimental groups $(\mathrm{n}=10$ /group): a control group of rats fed ad libitum with a standard rodent chow (4\% of energy from fat) (C), a HFD group with free access to a high-lipid content purified diet ( $45 \%$ of energy from fat), and another group subjected to energy restriction (75\% of daily individual intake of $\mathrm{C}$ rats) (ER). Metabolic and biometric parameters were evaluated as previously described [7]. Rats from each group were euthanized at 6 or 18 months $(n=5$ per age). Penis, heart and proximal portion of rat tail artery were removed, fixed in $10 \%$ buffered formalin and processed for imunohistochemical detection of $\alpha$ actin (specific SM cell marker) and Masson's trichrome staining by routine laboratory methods. All the slides were observed under an optical microscope coupled to a digital camera. The images captured were used to perform histomorphometric analysis (employing Image $^{\mathbb{R}}$ software) to evaluate $\mathrm{CC}$ and myocardium CT, as well as penile and tail artery SM contents. Statistical analysis was performed $\left(S P S S^{\circledR}\right.$ software) and statistical significance was assumed at $P<0.05$.

Masson's trichrome staining showed that CC and myocardium of HFD animals have a higher CT content compared to age-matched ER rats (Figure 1). Furthermore, the myocardium CT deposition occurs early in life in high-fat diet conditions. We also found that $\mathrm{CC}$ and heart collagen deposition is not age-dependent, as no differences were observed between 6 and 18-months-old $\mathrm{C}$ rats (Table 1). Also, either at 6 and 18-months, HFD led to a significant increase in corpora SM content, whereas ER animals presented just about half of that observed in $\mathrm{C} 18$-months rats. A significant increase in SM layer was also observed in CC of HFD fed rats during aging. No differences were observed in perivascular SM thickness of the rat tail artery.

Erectile function is tightly dependent of endothelial integrity. Growing evidence suggests that avoidance of HF consumption leads to early in life protection of endothelium, that not only prevents the onset of cardiovascular disease and/or the incidence of ED, but also, the physiologic effects of aging. Overall, our results highlight the outcomes of diet composition in different vascular beds, and reinforce the concept that structural changes in $\mathrm{CC}$ emerge before lesions of major organs as the heart 
in HFD conditions. Molecular studies are ongoing in order to clarify underlying mechanisms of age and diet-dependent structural changes in $\mathrm{CC}$ and myocardium of the rat.

\section{References}

1. Seo K. et al., J Androl 20: 298-306, 1999

2. Christ G., Urol Clin N Am 22: 727-745, 1995

3. Luo H. et al., J Sex Med 4: 644-645, 2007

4. Guay A., Endocrinol Metab Clin N Am 36: 453-463, 2007

5. Kaiser D. et al., J Am Coll Cardiol 43: 179-184, 2004

6. Ferrini M.G. et al., Nitric Oxide 6: 283-294, 2002

7. Tomada I. et al., Microsc Microanal 14 (suppl 3): 126-129, 2008

Table 1. Histomorphometric results from rat's CC and myocardium after Masson's Tricrome (CT content), and from rat tail artery and $\mathrm{CC}$ after $\alpha$-actin immunodetection (SM content). Values expressed as mean \pm SEM. Significant differences $(P<0.05)$ : (a) 6-months HFD versus 6-months ER; (b) 6-months $\mathrm{C}$ versus 6-months ER; (c) 6-months $\mathrm{C}$ versus 6-months HFD; (d) 6-months HFD versus 18-months HFD; (e) 18-months C versus 18-months HFD; (f) 18-months HFD versus 18-months ER.

\begin{tabular}{|c|c|c|c|c|c|c|c|}
\cline { 3 - 8 } \multicolumn{2}{c|}{} & \multicolumn{3}{c|}{ 6 months-old } & \multicolumn{3}{c|}{ 18 months-old } \\
\cline { 3 - 8 } \multicolumn{2}{c|}{} & C & HFD & ER & C & HFD & ER \\
\hline $\begin{array}{c}\text { Connective } \\
\text { tissue (\%) }\end{array}$ & Myocardium & $47.7 \pm 3.0$ & $54.3 \pm 2.5$ & $44.8 \pm 1.9^{(\mathrm{a})}$ & $50.2 \pm 2.4$ & $49.3 \pm 2.1$ & $46.7 \pm 2.5$ \\
\cline { 2 - 8 } & Cavernous tissue & $24.6 \pm 2.6$ & $33.8 \pm 2.8^{(\mathrm{c})}$ & $14.1 \pm 2.3^{(\mathrm{a}, \mathrm{b})}$ & $20.0 \pm 1.7$ & $45.5 \pm 2.7^{(\mathrm{d}, \mathrm{e})}$ & $24.0 \pm 3.5^{(\mathrm{f})}$ \\
\hline $\begin{array}{c}\text { Smooth } \\
\text { muscle (\%) }\end{array}$ & Rat tail artery & $35.1 \pm 1.3$ & $38.5 \pm 2.5$ & $41.4 \pm 2.1$ & $44.3 \pm 2.0$ & $39.5 \pm 0.8$ & $37.2 \pm 0.8$ \\
\cline { 2 - 8 } & Cavernous tissue & $9.9 \pm 0.9$ & $12.8 \pm 0.7^{(\mathrm{c})}$ & $7.8 \pm 0.4^{(\mathrm{a}, \mathrm{b})}$ & $12.6 \pm 1.3$ & $15.4 \pm 0.9^{(\mathrm{d}, \mathrm{e})}$ & $6.8 \pm 0.6^{(\mathrm{f})}$ \\
\hline
\end{tabular}

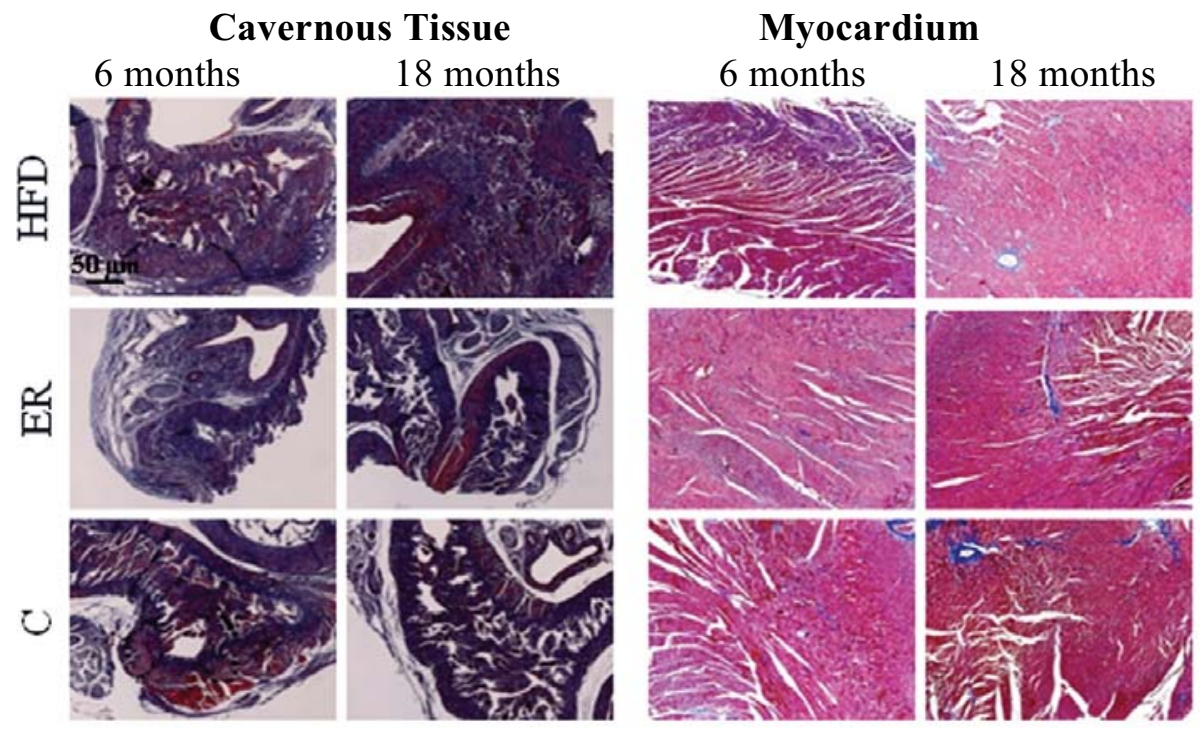

Figure 1. Masson's trichrome staining of rat's cavernous tissue and myocardium of all experimental groups. Smooth muscle and connective tissues are stained in red and in blue, respectively. 